\title{
LHCb status and overview
}

\section{Daria SAVRINA ${ }^{* \dagger}$}

M.V. Lomonosov Moscow State University and Institute of Theoretical and Experimental Physics

E-mail: Daria.Savrina@cern.ch

The LHCb detector provides a high efficiency for detection and reconstruction of decays of beauty particles produced in proton-proton collisions. Exploiting the power of the LHC machine during the past two years, LHCb has recorded $\sim 3 \mathrm{fb}^{-1}$ of data, which allowed to observe a number of new decays and increase the accuracy of previous measurements. An upgrade of both detector subsystems and trigger is planned for the $\mathrm{LHCb}$ in order to get ready for the increased instantaneous luminosity and bunch crossing frequency.

LHC on the March

20-22 November 2012

Institute for High Energy Physics, Protvino, Moscow region, Russia

\footnotetext{
*Speaker.

†n behalf of the LHCb collaboration.
} 


\section{Introduction}

The LHCb experiment is designed to study the properties of beauty and charmed particles decays. In such decays a search for the physics beyond the Standard Model (SM) is performed in an indirect way, as one expects the New Physics (NP) to contribute into the branching fractions of the rare decays or various asymmetries, like $C P$-asymmetry, isospin asymmetry, forward-backward asymmetry and many other observables. Heavy flavours provide more potentially interesting decays in this area. Large asymmetries are expected in $B$-mesons decays and their relatively long lifetimes give large opportunities for oscillation studies. Thus requiring a high agility and attentiveness such studies entice with a really great reward.

\section{2. $\mathrm{LHCb}$ detector at $\mathrm{LHC}$}

Among its other advantages the LHC has shown its ability to operate as a "flavour factory". The expected charm quark production rate in proton-proton collisions at $7 \mathrm{TeV}$ center-of-mass energy is $\sigma(p p \rightarrow c \bar{c} X) \sim 6 \mathrm{mb}$ [1] and for beauty quarks the production rate is $\sigma(p p \rightarrow b \bar{b} X) \sim$ $0.3 \mathrm{mb}[2]$. The produced $\mathrm{b}$ - and c-quarks hadronize in all possible beauty and charm species, which spread, predominantly, in a narrow cone around the beam axis. Of course, the rate of inelastic scattering processes is also high, thus an efficient trigger is essential for studies of the heavy flavours. Good mass resolution and particle identification are necessary for reduction of both combinatorial and peaking backgrounds. For the the studies of the effects showing up through $B_{s}^{0}-\bar{B}_{s}^{0}$ oscillations the propertime resolution should be comparable with the frequency of these oscillations $(\sim 350 \mathrm{fs})$.

These requirements set the constraints for the design of the $\mathrm{LHCb}$ detector geometry. The $\mathrm{LHCb}$ is a one-armed forward spectrometer [3], covering a solid angle $\sim 300 \mathrm{mrad}$, except for a small window around the beam-pipe. Such a geometry corresponds to a $2<\eta<5$ pseudorapidity range and ensures that $\sim 40 \%$ of all produced beauty and charmed particles enter the detector acceptance. The detector layout is shown in Fig. 1.

\subsection{VELO detector}

The VELO is a silicon-strip vertex detector, surrounding the $p p$ interaction region. It is constructed of two moving halves, each consisting of 21 modules, composed of one R-sensor and one $\phi$-sensor glued back-to-back. Each module is $220 \mu \mathrm{m}$ thick and has a radius of $120 \mathrm{~mm}$. During the data-taking the two halves are located at a distance of $0.7 \mathrm{~cm}$ from each other, but under unstable beam conditions they may be moved away from the beam to a distance of $6 \mathrm{~cm}$ from each other, in order to preserve the detector.

With 25 tracks the VELO detector has a spatial resolution of $\sim 16 \mu \mathrm{m}$ for $x$-and $y$-coordinates and $\sim 76 \mu \mathrm{m}$ for $z$-coordinate. For high transverse momentum tracks the resolution on the impact parameter with respect to the primary vertex is $20 \mu \mathrm{m}$. The decay time resolution is $\sim 45 \mathrm{fs}$, which provides an excellent possibility to distinguish between prompt particles and the ones produced in $b$-hadron decays. 


\subsection{Tracking system}

The tracking system includes four planes of silicon sensors (TT), $500 \mu \mathrm{m}$ thick each, a dipole magnet with a bending power of about $4 \mathrm{Tm}$ and three more tracking stations located downstream the magnet (T1-T3). TT is used mainly for reconstruction of tracks with low transverse momentum, which then loose all their energy in the magnet, and long-lived neutral particles, which decay outside the VELO detector. The inner part of the T1-T3 stations is made of silicon microstrip detectors, while the outer region, where the track multiplicities are not so high, is covered with straw tubes.

The track stations and the VELO detector together allow to reconstruct more than $96 \%$ of "long" tracks (that are the ones which start in VELO and end in the muon system) and provide momentum resolution from $0.4 \%$ for $\sim 5 \mathrm{GeV}$ energy tracks to $0.6 \%$ for $\sim 100 \mathrm{GeV}$ energy tracks.

\subsection{Ring-imaging Cherenkov detectors}

Charged hadrons are identified with two ring-imaging Cherenkov detectors (RICH1 and RICH2). RICH1 is located upstream the magnet right after the VELO detector, it uses two types of radiator: $\mathrm{C}_{4} \mathrm{~F}_{10}$ and aerogel for identification of charged hadrons in the $2 \mathrm{GeV} / \mathrm{c}<p_{T}^{h}<60 \mathrm{GeV} / \mathrm{c}$ transverse momenta range. The RICH2 detector is located downstream the magnet, $\mathrm{CF}_{4}$ radiator is used to identify the particles with momenta up to $100 \mathrm{GeV} / \mathrm{c}$. In both detectors the Cherenkov light is registered by hybrid photodiods with pixel readout. The whole system provides $95 \%$ efficiency in pion-kaon separation.

\subsection{Calorimeter system}

The calorimeter system includes four sub-detectors: scintillator pad detector (SPD), preshower detector (PRS), electromagnetic calorimeter (ECAL) and hadron calorimeter (HCAL). The SPD and PRS detectors consist of $\sim 6000$ scintillator pads each and are separated by a lead converter, whose thickness corresponds to 2.5 radiation lengths. The ECAL is built using the "shashlik"

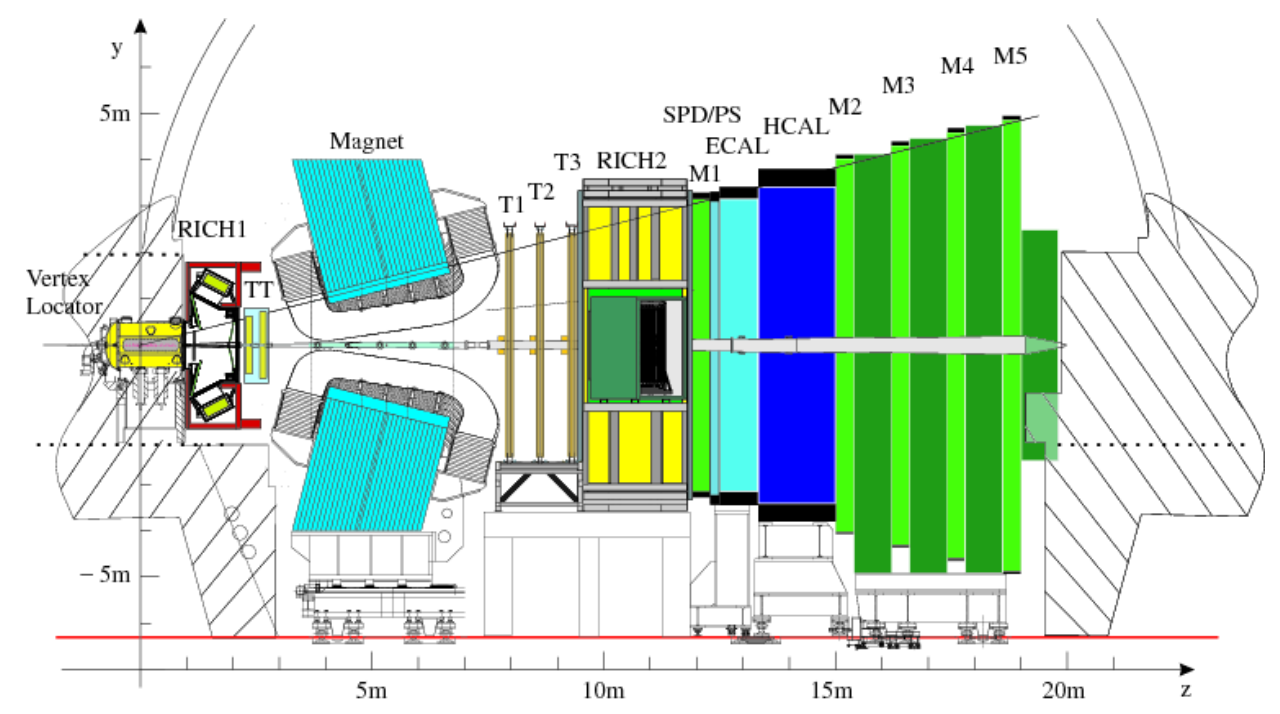

Figure 1: The LHCb detector layout. 
sampling technology. It consists of towers of alternating scintillator and lead tiles, having a total thickness of 25 radiation lengths. The HCAL is constructed of iron plates interspaced with scintillating tiles and is 5.6 interaction lengths thick. In all four subdetectors the scintillator light is collected by wavelength shifting fibers and read out with the multianode photomultipliers. The design energy resolution is $\frac{\sigma_{E}}{E(\mathrm{GeV})}=\frac{10 \%}{\sqrt{E}} \oplus 1 \%$ and $\frac{\sigma_{E}}{E(\mathrm{GeV})}=\frac{80 \%}{\sqrt{E}} \oplus 10 \%$ for ECAL and HCAL respectively.

The calorimeter system provides $\sim 90 \%$ efficiency in photon/electron/hadron separation and measures the energies of these particles with high accuracy. For example the mass resolution for radiative decays of the $B$-mesons is as good as $90 \mathrm{MeV} / \mathrm{c}^{2}$.

\subsection{Muon system}

The muon system includes five stations (M1-M5) of multi-wire proportional chambers and GEM detectors. The first station is located before the SPD, thus the calorimeter system acts as a muon filter between the first and second muon stations. For M2-M5 stations the muon filters are made of $80 \mathrm{~cm}$ iron plates, which corresponds to 20 interaction lengths. The system provides $\sim 97 \%$ efficiency for muon identification.

\subsection{Trigger}

The LHCb uses a three-step trigger: a hardware level-zero (L0) trigger is followed by a purely software high-level trigger, which is subdivided into two steps (HLT1 and HLT2). Relying on the information from calorimeter and muon systems L0 selects events with high transverse momentum photon, electron, hadron or muon candidates. HLT1, using information about reconstructed primary vertices and tracks, applies lifetime cuts, cuts on track momenta and impact parameters. At the HLT2 step the events are almost completely reconstructed and a number of exclusive and inclusive selections is run to retain only the events containing the decays of interest.

The whole trigger system reduces the initial $20 \mathrm{MHz}$ event rate to $3 \mathrm{kHz}(4.5 \mathrm{kHz}$ in 2012) level, at which the events are written to a storage. The trigger has an efficiency of $\sim 90 \%$ for dimuon channels, $\sim 30 \%$ for multibody hadronic final states and $\sim 10 \%$ for charm decays.

\subsection{Operation}

The $\mathrm{LHCb}$ experiment has started taking data from the very beginning of LHC operation in 2009. During last years the experiment was running at an instantaneous luminosity of $\mathscr{L}=$ $4 \times 10^{32} \mathrm{~cm}^{-2} \cdot \mathrm{s}^{-1}$ with a bunch crossing frequency of $20 \mathrm{MHz}$. About 1.5 primary vertices per bunch crossing are seen in the detector. This is almost four times higher than the value expected at the detector design stage, yet it did not prevent successful detector operation. The detector was fully operational $\sim 95 \%$ of the time and collected almost all the luminosity delivered by the LHC. The integrated luminosities collected by the LHCb experiment in each year are listed in Table 1.

In 2012 LHCb had an exciting experience of working with proton-ion collisions. The multiplicities seen in the detector during such runs were comparable with the ones obtained in the proton-proton collisions and the detector was showing a smooth and stable behavior. Thus a decision was made to take part in proton-ion runs in the beginning of 2013 for which various production and polarization studies are planned. 
Table 1: Integrated luminosities collected by the LHCb experiment.

\begin{tabular}{ccc} 
Year & $\begin{array}{c}\text { Center-of-mass } \\
\text { energy }\end{array}$ & $\begin{array}{c}\text { Integrated } \\
\text { luminosity }\end{array}$ \\
\hline 2010 & $7 \mathrm{TeV}$ & $37 \mathrm{pb}^{-1}$ \\
2011 & $2.76 \mathrm{TeV}$ & $71 \mathrm{pb}^{-1}$ \\
2011 & $7 \mathrm{TeV}$ & $1.0 \mathrm{fb}^{-1}$ \\
2012 & $8 \mathrm{TeV}$ & $1.8 \mathrm{fb}^{-1}$
\end{tabular}

\section{Highlights of recent physics results}

The physics program of the $\mathrm{LHCb}$ experiment covers a wide number of topics, among which not only the studies of beauty and charm decays, but also searches for exotic particles and decays. Recent results obtained for the most promising studies are given below.

\subsection{Mixing-induced $C P$-violation in the $B_{s}^{0}$ system}

The value of the mixing induced $C P$-violation in the $B_{s}^{0}$ system is governed by the $B_{s}^{0}$-mesons mixing phase $\phi_{S}$. Within the SM the value of this phase is predicted with a very good accuracy via indirect measurements and is expected to be very small: $\phi_{s}=-2 \arg \left(-V_{t s} V_{t b}^{*} / V_{c s} V_{c b}^{*}\right)=-0.036 \pm$ 0.002 [5]. The NP contributions may induce large deviations from this value, which would become immediately visible.

The value of $\phi_{s}$ may be measured through a variety of $B_{s}^{0}$ decay modes, including the "golden mode", the $B_{s}^{0} \rightarrow J / \psi \phi$ decay. Compared to the $B^{0}$, the $B_{s}^{0}$ oscillate much faster and the $\Delta \Gamma_{s} \equiv$ $\Gamma_{L}-\Gamma_{H}$ value, which is the mean decay width difference between the light and heavy states, is not negligible and can be measured as well.

As $J / \psi \phi$ is not a $C P$-eigenstate an angular analysis was performed for this decay in order to separate the $C P$-even and $C P$-odd contributions. It has also been observed for the first time, that $\Delta \Gamma_{s}$ has a non-zero value. However, since the time-dependent differential decay rates are invariant under the simultaneous replacement $\left(\phi_{s}, \Delta \Gamma_{s}\right) \rightarrow\left(\pi-\phi_{s},-\Delta \Gamma_{s}\right)$, the sign of $\Delta \Gamma_{s}$ should also be determined in order to select one of the solutions for $\phi_{s}$. This ambiguity has been solved a bit earlier by studying the behavior of the difference between $\mathrm{S}$-wave and $\mathrm{P}$-wave phases in a wide $K^{+} K^{-}$invariant mass window [6]: $988 \mathrm{MeV} / \mathrm{c}^{2}<M\left(K^{+} K^{-}\right)<1050 \mathrm{MeV} / \mathrm{c}^{2}$. With $4.7 \sigma$ significance it has been shown that the $\Delta \Gamma_{s}>0$ solution demonstrates the expected behavior [7], i.e. a heavy $B_{s}^{0}$ state lives longer, than the light one. As the result of a global fit the values of the mixing phase, mean decay widths difference and the decay width were found to be:

$$
\begin{aligned}
\phi_{s} & =-0.001 \pm 0.101 \pm 0.027 \mathrm{rad} \\
\Delta \Gamma_{s} & =\left(\begin{array}{ll}
0.116 \pm 0.018 \pm 0.006
\end{array}\right) \mathrm{ps}^{-1} \\
\Gamma_{s} & =(0.6580 \pm 0.0054 \pm 0.0066) \mathrm{ps}^{-1}
\end{aligned}
$$

where the first uncertainty is statistical and the second is systematic [4]. This is currently the most precise measurement of these values and $\phi_{s}$ and $\Delta \Gamma_{s}$ show no deviation from the SM prediction (see Fig. 2). 


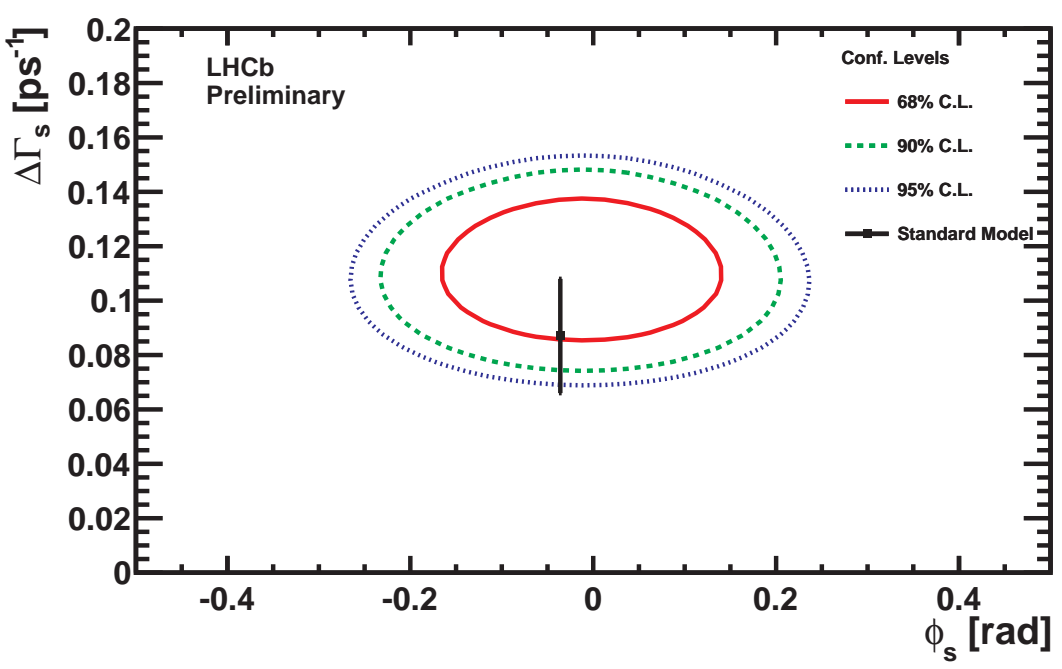

Figure 2: Confidence regions in the $\phi_{s}-\Delta \Gamma_{s}$ plane. Only statistical uncertainties are included. The black square corresponds to the theoretically predicted Standard Model value.

Another channel, used at $\mathrm{LHCb}$ for the measurement of the $B_{s}^{0}$ mixing phase is the $B_{s}^{0} \rightarrow$ $J / \psi \pi^{+} \pi^{-}$decay. For this study the dipion invariant mass window was selected to be $775 \mathrm{MeV} / \mathrm{c}^{2}<$ $M\left(\pi^{+} \pi^{-}\right)<1550 \mathrm{MeV} / \mathrm{c}^{2}$. It has been shown [8], that the $J / \psi \pi^{+} \pi^{-}$final state in this region is dominated by CP-odd S-wave by more than $97.7 \%$ and thus no angular analysis was performed. The value of the mixing phase was found to be $\phi_{s}=-0.02 \pm 0.17 \pm 0.02 \mathrm{rad}$, where the first uncertainty is statistical and the second is systematic [9].

The combined result for the $\phi_{s}$ measured in $B_{s}^{0} \rightarrow J / \psi \phi$ and $B_{s}^{0} \rightarrow J / \psi \pi^{+} \pi^{-}$decays is $\phi_{s}=$ $-0.002 \pm 0.083 \pm 0.027$, where the first uncertainty is statistical and the second is systematic. This value is in agreement with the SM prediction. Several more channels, like $B_{s}^{0} \rightarrow J / \psi \eta^{(\prime)}$ [10] and first time observed $B_{s}^{0} \rightarrow J / \psi \mathrm{f}_{2}^{\prime}(1525)$ [11], are being studied for the additional independent measurements of the mixing phase.

\subsection{Measurement of the $\gamma$ angle}

The $\gamma$ angle of the Unitarity Triangle (UT) may be defined through the CKM-matrix elements as the following: $\gamma=\arg \left(V_{u d} V_{u b}^{*} / V_{c d} V_{c b}^{*}\right)$. Among the other sides and angles of the UT this one is the least-well determined with current uncertainties of the direct measurements about 10-12 $[5$, 12].

It has been proven [13] that the cleanest method for measurement of the $\gamma$ angle is through the study of $B^{ \pm} \rightarrow D h^{ \pm}$decays, where $h^{ \pm}$corresponds to either $K^{ \pm}$- or $\pi^{ \pm}$-meson and $D$ denotes either $D^{0}$ or $\overline{D^{0}}$. The $D$ decay mode should be accessible for both $D^{0}$ and $\overline{D^{0}}$.

LHCb has already presented results of measurements of the $\gamma$ angle in $B^{ \pm} \rightarrow D h^{ \pm}$decays followed by various $D$-meson decay modes obtained with $1 \mathrm{fb}^{-1}$ of data $[14,15,16]$. Many of the used modes, namely $B^{ \pm} \rightarrow\left[\pi^{ \pm} K^{\mp}\right]_{D} K^{ \pm}, B^{ \pm} \rightarrow\left[\pi^{ \pm} K^{\mp} \pi^{+} \pi^{-}\right]_{D} K^{ \pm}$and $B^{ \pm} \rightarrow\left[\pi^{ \pm} K^{\mp} \pi^{+} \pi^{-}\right]_{D} \pi^{ \pm}$, were observed for the first time. Using these results a global fit has been performed. First, the available observables of the $D K$ system, for which the interference and thus the sensitivity is expected to be very high, were combined (see Fig. 3(a)). It resulted in a $\gamma$ angle value of $\gamma=\left(71.1_{-15.7}^{+16.6}\right)^{o}$. Then, 

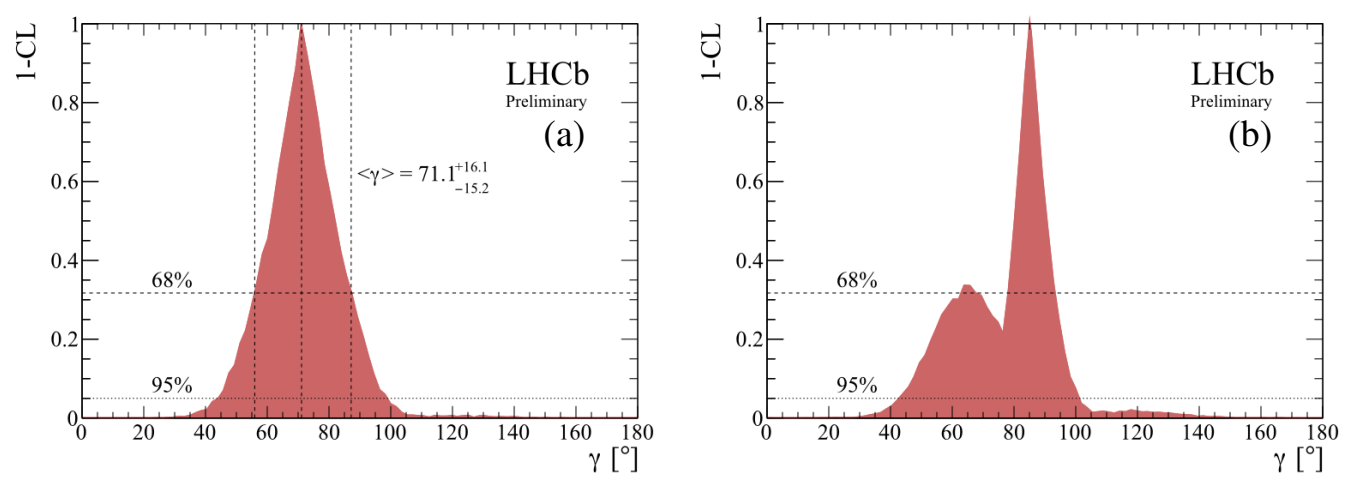

Figure 3: $1-C L$ curve for $\gamma$ (a) for the combination of the $D K$ measurements; (b) the full $D K$ and $D \pi$ combination.

for the first time, the information about the $B^{ \pm} \rightarrow D \pi^{ \pm}$was included in the combination (Fig. 3(b)) and the best-fit value became $\gamma=85.1^{\circ}$ with confidence limits $\gamma \in[43.8,101.5]^{\circ} @ 95 \%$ CL [17].

\section{3 $B_{(s)}^{0} \rightarrow \mu^{+} \mu^{-}$decays}

One of the most exciting studies performed by the LHCb experiment, which gave result in 2012 , is the search for the very rare $B_{(s)}^{0} \rightarrow \mu^{+} \mu^{-}$decays. In the SM the branching fractions of these decays are predicted with a very good accuracy: $\mathrm{BR}\left(\mathrm{B}_{\mathrm{s}}^{0} \rightarrow \mu^{+} \mu^{-}\right)=(3.23 \pm 0.27) \times 10^{-9}$ and $\mathrm{BR}\left(\mathrm{B}^{0} \rightarrow \mu^{+} \mu^{-}\right)=(0.107 \pm 0.010) \times 10^{-9}[18]$, thus any excess above the predicted value should be immediately visible and mean the NP contribution. For example, these decays are predicted to be very sensitive to the models with an extended Higgs sector and high $\tan \beta$.

At $\mathrm{LHCb}$ a search for the $B_{(s)}^{0} \rightarrow \mu^{+} \mu^{-}$decays is performed through a 2-dimensional analysis in bins of boosted decision tree (BDT) and dimuon invariant mass. The BDT is constructed of topological variables uncorrelated with the invariant mass. The signal mass region was blinded during the analysis.

With $1 \mathrm{fb}^{-1}$ of data collected by the LHCb in 2011 the tightest upper limits were set at $95 \% \mathrm{CL}$ for both decays: $\mathrm{BR}\left(\mathrm{B}_{\mathrm{s}}^{0} \rightarrow \mu^{+} \mu^{-}\right)<4.5 \times 10^{-9}$ and $\mathrm{BR}\left(\mathrm{B}^{0} \rightarrow \mu^{+} \mu^{-}\right)<1.03 \times 10^{-9}$ [19], which for $B_{s}^{0}$ decay was already very close to the SM prediction. After adding a $1.1 \mathrm{fb}^{-1}$ datasample, collected in 2012, the first evidence of the $B_{s}^{0} \rightarrow \mu^{+} \mu^{-}$decay was found with a statistical significance of $3.5 \sigma$ (see Fig. 4). The branching fraction of this decay was measured to be $\mathrm{BR}\left(\mathrm{B}_{\mathrm{s}}^{0} \rightarrow \mu^{+} \mu^{-}\right)=$ $\left(3.2_{-1.2-0.3}^{+1.4+0.5}\right) \times 10^{-9}$ [20], where the first uncertainty is statistical and the second one is systematic. For the $B^{0} \rightarrow \mu^{+} \mu^{-}$decay a tighter upper limit was set: $\mathrm{BR}\left(\mathrm{B}^{0} \rightarrow \mu^{+} \mu^{-}\right)<0.94 \times 10^{-9} @ 95 \%$ CL. These measurements strongly constrain TeV-scale SUSY models, but there is still space for the other models, which behave similar to the SM in this point.

\subsection{The $B^{0} \rightarrow K^{*} \mu^{+} \mu^{-}$decay}

One more example of a rare decay which attracts a special interest is the $B^{0} \rightarrow K^{*} \mu^{+} \mu^{-}$ decay. It proceeds through flavour-changing neutral current (FCNC) loop diagrams with a $b \rightarrow s$ quark transition. Detailed studies of angular distributions in this decay may give an access to NP phenomena. A special sensitivity is expected in study of the shape of forward-backward asymmetry 


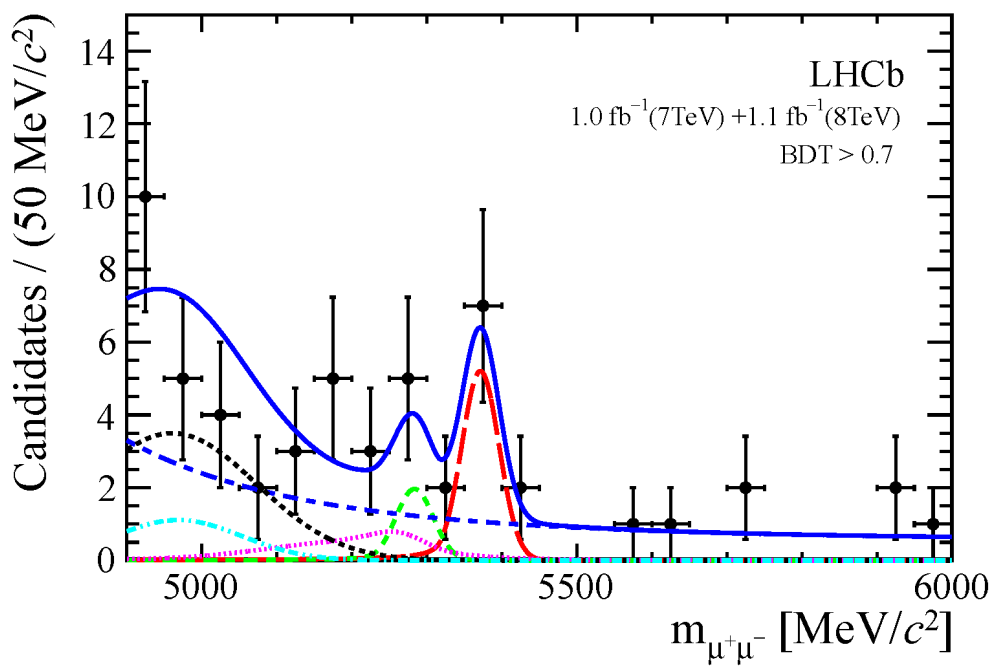

Figure 4: Invariant mass distribution of the selected $B_{s}^{0} \rightarrow \mu^{+} \mu^{-}$candidates (black dots) with BDT $>0.7$ in the combined 2011+2012 dataset. The fit function (solid blue line) and its components are described in [20].

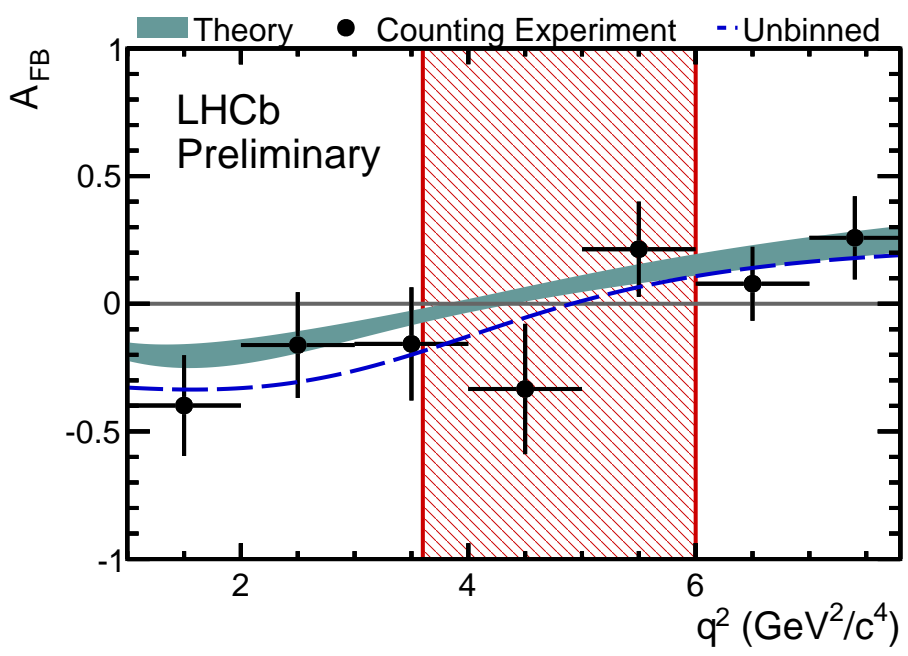

Figure 5: The $A_{\mathrm{FB}}$ as a function of $q^{2}$, that comes from the unbinned counting experiment (blue dashed line) overlaid with the theory prediction from Ref. [21]. The data-points are the result of counting forward- and backward-going events in $1 \mathrm{GeV}^{2} / c^{4}$ bins of $q^{2}$. The uncertainty on the data-points is statistical only. The red-hatched region is the $68 \%$ confidence interval on the zero-crossing point observed in the data.

$\left(A_{\mathrm{FB}}\right)$ as a function of the dimuon invariant mass squared $q^{2}$ and the point at which the $A_{\mathrm{FB}}$ changes its sign, the zero-crossing point. The $q^{2}$ of this point is predicted to lie within the $[4,4.3] \mathrm{GeV}^{2} / \mathrm{c}^{4}$ range. With $1 \mathrm{fb}^{-1}$ of data a clean sample of $900 \pm 32 B^{0} \rightarrow K^{*} \mu^{+} \mu^{-}$events was collected and the position of the zero-crossing point was measured to be $q^{2}\left(A_{\mathrm{FB}}=0\right)=4.9_{-1.3}^{+1.1} \mathrm{GeV}^{2} / \mathrm{c}^{4}$ [22] (see Fig. 5), which is very well consistent with the SM prediction. 


\subsection{Radiative decays of $B$-mesons}

Another example of FCNC loop processes involving a $b \rightarrow s$ quark transition are the radiative decays $B^{0} \rightarrow K^{*} \gamma$ and $B_{s}^{0} \rightarrow \phi \gamma$. These decays are not as rare as the previous ones, their branching fractions are predicted to be $(4.3 \pm 1.4) \times 10^{-5}$ for both decays [23] and the $B^{0} \rightarrow K^{*} \gamma$ decay has been observed for the first time already in 1993 by the CLEO collaboration [24].

Theoretical predictions for the branching fractions of these decays suffer from large unavoidable uncertainties raised by the hadronic form-factors. However, the radiative decays provide a number of other interesting observables in which these uncertainties cancel out [25]. Among them are the ratio of branching fractions $\frac{\mathrm{BR}\left(\mathrm{B}^{0} \rightarrow \mathrm{K}^{*} \gamma\right)}{\mathrm{BR}\left(\mathrm{B}_{\mathrm{s}}^{0} \rightarrow \phi \gamma\right)}=1.0 \pm 0.2$ and the direct $C P$-asymmetry in $B^{0} \rightarrow K^{*} \gamma$ decay, which is predicted to be $A_{C P}\left(B^{0} \rightarrow K^{*} \gamma\right)=(-0.61 \pm 0.43) \%$.

Large signal samples for both decays were collected by LHCb with $1 \mathrm{fb}^{-1}$ of data: $N_{B^{0} \rightarrow K^{*} \gamma}=$ $5279 \pm 93$ and $N_{B_{s}^{0} \rightarrow \phi \gamma}=691 \pm 36$ [26]. The ratio of branching fractions of these two decays was measured to be $\frac{\mathrm{BR}^{\circ}\left(\mathrm{B}^{0} \rightarrow \mathrm{K}^{*} \gamma\right)}{\mathrm{BR}\left(\mathrm{B}_{\mathrm{s}}^{0} \rightarrow \phi \gamma\right)}=1.23 \pm 0.06 \pm 0.04 \pm 0.01$, where the first uncertainty is statistical, the second one is systematic and the third one is due to the uncertainty of the b-quark hadronization fractions ratio. The direct $C P$-asymmetry in the $B^{0} \rightarrow K^{*} \gamma$ decay was found to be $A_{C P}\left(B^{0} \rightarrow K^{*} \gamma\right)=$ $(0.8 \pm 1.7 \pm 0.9) \%$, where the first uncertainty is statistical and the second is systematic. Both measurements are currently the most precise ones and show no deviation from the SM predictions.

\section{Upgrade}

In the upcoming years a lot of work is planned towards the LHC upgrade. LHCb is also getting ready for new conditions of data taking with an instantaneous luminosity of $10^{33} \mathrm{~cm}^{-2} \cdot \mathrm{s}^{-1}$ and a $40 \mathrm{MHz}$ bunch crossing frequency. In 2011 the Letter of Intent [27] was presented, in which the upgrade motivation and main points are described. The evolution of the research and development (R\&D) and the detailed timescale are given in the Framework TDR [28], published in 2012. The subsystem TDRs are expected to be ready in 2013. In parallel with this the R\&D studies are ongoing. During the technical stop in 2013-2014 the first infrastructures for upgrade are going to be produced and installed. The detector upgrade is planned for 2018 technical stop, after which $\mathrm{LHCb}$ is going to collect $\sim 5 \mathrm{fb}^{-1}$ per year.

The main changes in the detector are planned for the tracking system (including VELO), where the replacement of all the silicon sensors is going to be done. For both RICH detectors and the calorimeter system the photomultipliers are going to be replaced. It was also decided to make the trigger purely software, thus making it faster and more efficient under higher occupancies. It is expected that the trigger will be provided with an access to the full event information and this requires a replacement of all the front-end electronics to be able to readout at $40 \mathrm{MHz}$.

The LHCb awaits a lot of both "exploration", which means the search for the not yet seen effects and decays, and "precision" studies, i.e. increase of the accuracy of measurements of known phenomena. The expected sensitivities of the upgraded LHCb detector may be found in Ref. [27]. With $50 \mathrm{fb}^{-1}$ of data for most of promising measurements the precision is expected to reach the order of the theoretical predictions accuracy. 


\section{Conclusions}

The LHCb detector demonstrates a brilliant shape, which allows to make lots of most precise measurements and first observations in already first year of data taking. The evidence of the rarest decay seen by the moment, the $B_{s}^{0} \rightarrow \mu^{+} \mu^{-}$decay, was found with only 1.5 years of data taking. Unfortunately the New Physics did not show up yet, but there is still a lot of space for it left and better precision is needed. The LHCb has big plans for the upgrade and is looking forward for more data and new perspectives.

\section{References}

[1] LHCb Collaboration, Prompt charm production in pp collisions at $\sqrt{s}=7 \mathrm{TeV}$, LHCb-CONF-2010-013

[2] LHCb Collaboration, Measurement of $\sigma(p p \rightarrow b \bar{b} X) a t \sqrt{s}=7 \mathrm{TeV}$ in the forward region, Phys. Lett. B694 (2010) 209-216

[3] LHCb Collaboration, The LHCb Detector at the LHC, JINST 3, S08005 (2008)

[4] LHCb Collaboration, Tagged time-dependent angular analysis of $B_{s}^{0} \rightarrow J / \psi \phi$ decays at LHCb, LHCb-CONF-2012-002

[5] J. Charles et al. (CKMfitter group), Predictions of selected flavor observables within the standard model, Phys. Rev. D84 (2011) 033005

[6] Y.Xie et al., Determination of $2 \beta_{s}$ in $B_{s}^{0} \rightarrow J / \psi K^{+} K^{-}$decays in the presence of a $K^{+} K^{-} S$-wave contribution, JHEP 0909, 074 (2009)

[7] LHCb Collaboration, Determination of the sign of the decay width difference in the $B_{s}^{0}$ system, Phys. Rev. Lett. 108, 241801 (2012)

[8] LHCb Collaboration, Analysis of the resonant components in $\overline{B_{s}^{0}} \rightarrow J / \psi \pi^{+} \pi^{-}$, Phys. Rev. D86, 052006 (2012)

[9] LHCb Collaboration, Measurement of the CP-violating phase $\phi_{s}$ in $\overline{B_{s}^{0}} \rightarrow J / \psi \pi^{+} \pi^{-}$decays, Phys. Rev. B713, 378-386 (2012)

[10] LHCb Collaboration, Evidence for the decay $B^{0} \rightarrow J / \psi \omega$ and measurement of the relative branching fractions of $B_{s}^{0}$ meson decays to $J / \psi \eta$ and $J / \psi \eta^{\prime}$, arXiv:1210.2631 (2012)

[11] LHCb Collaboration, Observation of $\overline{B_{s}^{0}} \rightarrow J / \psi f_{2}^{\prime}(1525)$ in $J / \psi K^{+} K^{-}$final states, Phys. Rev. Lett. 108, 151801 (2012)

[12] M. Bona et al., The 2004 UTfit collaboration report on the status of the unitarity triangle in the Standard Model, JHEP 0507 (2005) 028, arXiv:hep-ph/0501199.

[13] M. Gronau, D. Wyler, On determining a weak phase from charged B decay asymmetries, Phys. Lett. B265, 172 (1991)

[14] LHCb Collaboration, Observation of the CP-violation in $B^{ \pm} \rightarrow D K^{ \pm}$decays, Phys. Lett. B712 (2012) 203

[15] LHCb Collaboration, A model-independent Dalitz plot analysis of $B^{ \pm} \rightarrow D K^{ \pm}$with $D \rightarrow K_{S}^{0} h^{+} h^{-}(h=\pi, K)$ decays and constraints on the CKM angle $\gamma$, Phys. Lett. B718 (2012) 43-55

[16] LHCb Collaboration, First observation of the suppressed ADS modes $B^{ \pm} \rightarrow\left[\pi^{ \pm} K^{\mp} \pi^{+} \pi^{-}\right]_{D} K^{ \pm}$and $B^{ \pm} \rightarrow\left[\pi^{ \pm} K^{\mp} \pi^{+} \pi^{-}\right]_{D} \pi^{ \pm}$, LHCb-CONF-2012-030 (2012) 
[17] LHCb Collaboration, A measurement of $\gamma$ from a combination of $B^{ \pm} \rightarrow D h^{ \pm}$analyses, LHCb-CONF-2012-032 (2012)

[18] A. J. Buras, J. Girrbach, D. Guadagnoli, and G. Isidori, On the standard model prediction for $\mathrm{B}\left(\mathrm{B}_{\mathrm{s}, \mathrm{d}} \rightarrow \mu^{+} \mu^{-}\right)$, Eur. Phys. J. C72 (2012) 2172

[19] LHCb Collaboration, Strong constraints on the rare decays $B_{s} \rightarrow \mu^{+} \mu^{-}$and $B_{d} \rightarrow \mu^{+} \mu^{-}$, Phys. Rev. Lett. 108, 231801 (2012)

[20] LHCb Collaboration, First evidence for the decay $B_{s}^{0} \rightarrow \mu^{+} \mu^{-}$, Phys. Rev. Lett. 110, 021801 (2013)

[21] C. Bobeth, G. Hiller, and D. van Dyk, More Benefits of Semileptonic Rare B Decays at Low Recoil: CP Violation, arXiv:1105.0376, see also references therein.

[22] LHCb Collaboration, Differential branching fraction and angular analysis of the $B^{0} \rightarrow K^{* 0} \mu^{+} \mu^{-}$ decay, LHCb-CONF-2012-008 (2012)

[23] A. Ali, B.D. Pecjak, C. Greub, Towards B $\rightarrow V \gamma$ decays at NNLO in SCET, Eur. Phys. J. C55, 577-595 (2008)

[24] R. Amar et al., CLEO Collaboration Evidence for penguins: First observation of $B \rightarrow K^{*}(892) \gamma$, Phys.Rev.Lett. 71 (1993) 674-678

[25] M. Matsumori, A.I. Sanda, Y.-Y. Keum, CP asymmetry, branching ratios and isospin breaking effects of $B \rightarrow K^{*} \gamma$ with the perturbative QCD approach, Phys.Rev. D72 (2005) 014013

[26] LHCb Collaboration, Measurement of the ratio of branching fractions $\mathscr{B}\left(B^{0} \rightarrow K^{* 0} \gamma\right) / \mathscr{B}\left(B_{s}^{0} \rightarrow \phi \gamma\right)$ and the direct $C P$ asymmetry in $B^{0} \rightarrow K^{* 0} \gamma$, Nucl. Phys. B867 (2012) 1-18

[27] LHCb Collaboration, Letter of Intent for the LHCb Upgrade, CERN-LHCC-2011-001 (2011)

[28] LHCb Collaboration, Framework TDR for the LHCb upgrade, CERN-LHCC-2012-007 (2012) 